\title{
Proyecto Dorothy: propuestas de innovación en la logística urbana. El Joint Action Plan
}

\author{
$\mathbf{M}^{\mathrm{a}}$ Isabel Espinós Garcia \\ Resp. Proyectos, Movilidad Urbana Sostenible, España \\ Marco Mastreta \\ CEO, T3 srl, Italia \\ Alessandro Monti \\ Senior Project Manager, EU Project Manager, Fondazione per la Ricerca e l'Innovazione \\ promossa dall’Università di Firenze e la Città Metropolitana di Firenze
}

\section{RESUMEN}

El proyecto DOROTHY "Development Of RegiOnal clusTers for researcH and implementation environmental friendlY urban logistics" (www.clusterdorothy.com), financiado por la Comisión Europea dentro del programa Regiones del Conocimiento se ha centrado en la creación de un marco que favorezca la innovación en el sector de la logística urbana.

Como uno de los principales resultados del proyecto se han creado 3 clústeres a nivel regional en la Comunidad Valenciana, la región del Valle del Tajo y Lisboa en Portugal y la región de Oltenia en Rumanía, en linea con el clúster de logística urbana ya existente en la región de Toscana en Italia y que ha servido de ejemplo. En colaboración con estos clústeres se ha redactado el Joint Action Plan (JAP) que comprende herramientas para potenciar la innovación y propuestas concretas para poner en práctica en el corto y medio plazo.

Esta ponencia resume los contenidos del JAP.

\section{INTRODUCCIÓN}

\subsection{Antecedentes}

El desafío de lograr que la logística urbana sea más sostenible se ha convertido en un elemento de discusión y análisis común en ciudades de todo el mundo. De hecho, se trata de un desafío crítico si tenemos en cuenta que alrededor del $75 \%$ de los europeos viven en ciudades y que se espera que este porcentaje alcance el $82 \%$ en 2050. Además, las operaciones relacionadas con la Logística urbana tienen un impacto importante sobre la movilidad y el consumo de energía, al tiempo que resultan indispensables para el propio funcionamiento de la ciudad.

Para alcanzar esa sostenibilidad en la logística urbana se requiere un esfuerzo innovador en 
el que participen administraciones y organismos públicos, operadores, agentes económicos, desarrolladores de tecnología y departamentos de I + D. De hecho, el propio sector de la logística urbana representa un mercado de referencia para la aplicación de nuevas tecnologías de la información, nuevos vehículos, envases, sistemas de automatización de almacenes, plataformas de apoyo, etc. que supone un incentivo para las empresas del sector.

En este sentido, el JAP define estrategias, tanto para entes públicos como para empresas, de cara a incrementar y potenciar la innovación del sector y la cooperación entre las regiones, a través de los clústeres, que forman parte del proyecto Dorothy.

\subsection{Objetivos del JAP}

El JAP tiene como objetivo definir un plan de acción para abordar las cuestiones fundamentales de la logística urbana según los objetivos estratégicos definidos en propio proyecto Dorothy. El plan establece una priorización de las acciones y las necesidades de cooperación entre los agentes implicados, tratando de sacar el mayor provecho de las capacidades de cada región del proyecto Dorothy. Como elemento clave, se incluye también un marco de referencia para la financiación de cada acción así como una estimación del coste de puesta en marcha y del mercado potencial.

Para transferir adecuadamente e implementar las soluciones innovadoras de I + D que se plantean, en la mayoría de los casos se requiere el apoyo y la coordinación de los distintos agentes del sector teniendo en cuenta que se afecta los siguientes marcos: legal y regulatorio, la tecnología, la gestión del cambio, la financiación y los resultados de la evaluación y difusión.

\subsection{Metodología del JAP.}

Teniendo en cuenta la gran y urgente necesidad de innovación y desarrollo que tiene la logística urbana, seleccionar las prioridades y, posteriormente, las acciones de mayor interés, ha supuesto un reto para el proyecto.

Para establecer las prioridades del JAP se estableció un enfoque que podemos llamar de fusión y combinación de acciones complementarias en materias concretas. Los criterios para seleccionar las acciones se basaron en

- La facilidad de implantar nuevos productos / servicios sobre la base de la excelencia

- Promover la investigación colaborativa y el desarrollo tecnológico entre las regiones europeas

- Mejorar la transferencia de conocimiento de los departamentos de I + D hacia las empresas, con especial atención a las Pymes, a través de redes que cuenten con el apoyo de los clústeres regionales. 
- Mejorar el acceso a la financiación e incentivos públicos para la innovación;

- Promover la especialización a nivel regional

- Desarrollar el potencial de creación de redes de contactos a nivel Europeo y la internacionalización de productos y servicios para el sector.

Para hacer frente a los desafíos y las prioridades definidas, se estructuraron una serie de áreas temáticas para cada uno de los aspectos específicos del proceso general de la logística urbana con el fin de organizar las acciones del JAP:

- Políticas y Regulaciones

- Evaluación y Estandarización

- Desarrollo Regional de los Clústeres

- Formación

- Planeamiento urbanístico

- Innovación y la cooperación científica

- Internacionalización de los Clústeres.

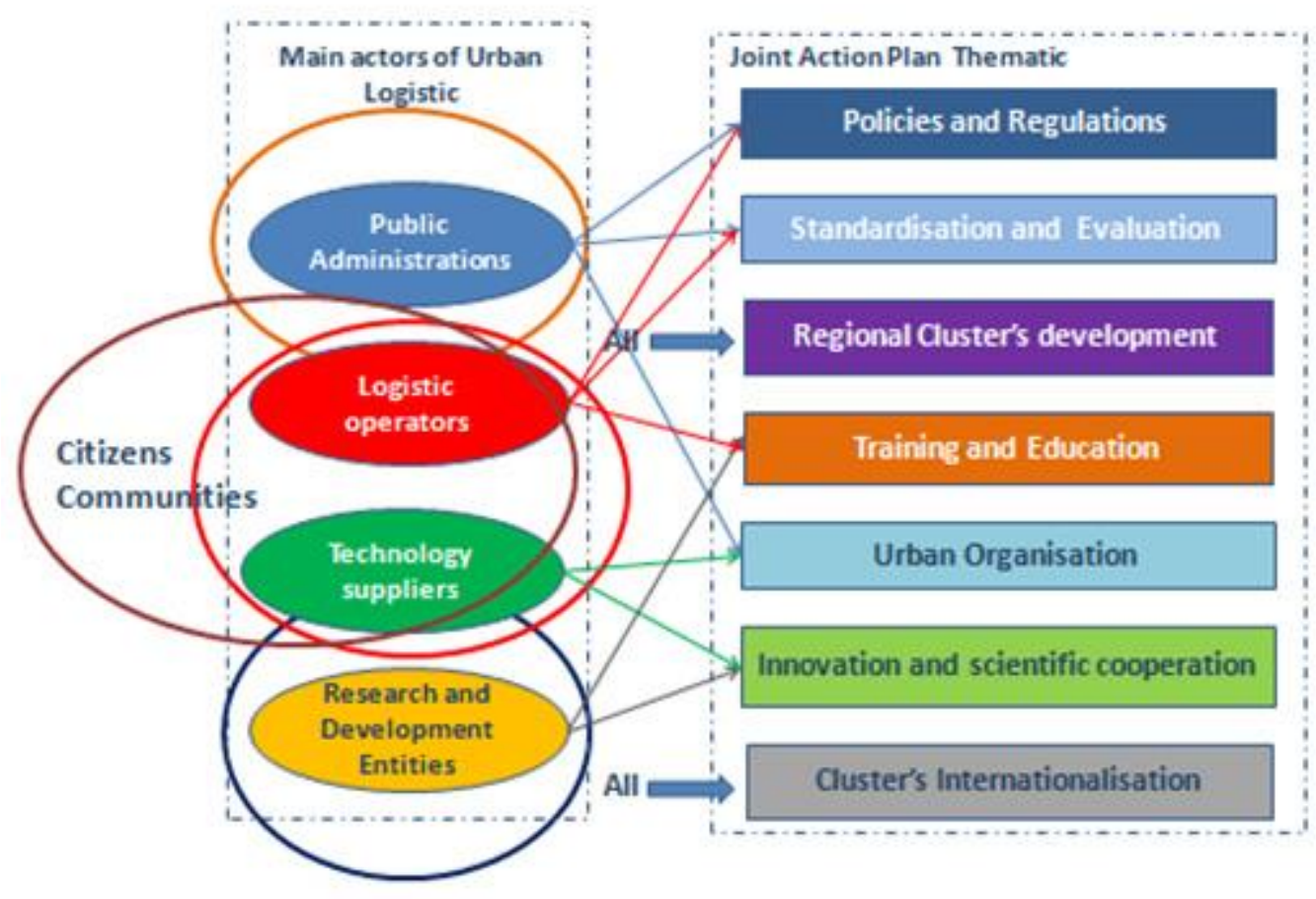

Fig. 1 - Áreas temáticas y agentes implicados.

El Proyecto Dorothy ha trabajado en el JAP entre 2013 y 2016, involucrando de forma activa a los agentes del sector, principalmente a través de los Clústeres, por lo que las acciones están orientadas hacia ellos tratando de sacar provecho en cada región de las capacidades y potenciales locales.

\section{LAS REGIONES: PROBLEMAS Y OPORTUNIDADES DE LA LOGÍSTICA}




\section{URBANA.}

Dadas las diferencias existentes entre las regiones analizadas, en términos de estructura urbana, desarrollo económico y necesidades logísticas, el JAP se ha diseñado con el fin de satisfacer necesidades diferentes, con distintos niveles de interés de unas regiones en determinadas acciones. Por otra parte, todas las acciones se han diseñado con el objetivo desarrollar un alto potencial de replicación en otras ciudades europeas.

\subsection{Estructura del mercado.}

Desde el punto de vista de la innovación tecnológica el campo de la logística urbana es un conglomerado que agrupa distintas tecnologías, competencias y aplicaciones entre las que podemos citar:

- Ingeniería, relacionado principalmente con el modelado, simulación y diseño de nuevos modelos y metodologías de evaluación.

- Tecnología de la información para la gestión de flotas y mercancías, la planificación y la programación, gestión de la información en tiempo real, entre otros.

- Equipos electrónicos, como son los equipos embarcados para el seguimiento, planificación, comunicación y aplicaciones en tiempo real.

- Vehículos respetuosos con el medio ambiente

- Mecánica y mecatrónica, relativa a los envases y material de mantenimiento, equipos y soluciones de almacén, sistemas de carga / descarga, etc.

De acuerdo con lo anterior, el JAP desarrolla una segmentación del mercado que facilita después su integración y consideración en las acciones. Muy sucintamente, la segmentación considerada ha sido la siguiente.

- Sistemas de gestión de la distribución de las mercancías: generalmente nos referimos a los sistemas de TIC que dan la posibilidad de gestionar todas las operaciones de distribución y almacén. Ellos pueden diferenciarse en varias clases:

- Hardware específico para la gestión de la distribución.

- Software específico para sistemas de distribución de mercancías.

- Sistemas de apoyo a los regímenes de regulación. Entre estos sistemas podemos mencionar los dedicados especialmente a la distribución de la carga o que están estrechamente relacionadas con ella, como los sistemas de control de accesos.

- Sistemas de almacenamiento automático y sistemas de manipulación. Dentro de esta categoría, que incluye los sistemas multi-tecnológicos (mecánica, electrónica y TIC), podemos considerar tanto el equipo para el almacenamiento, como para la carga y descarga: sistemas de almacenaje, de manipulación y sistemas de picking, etc.

- Sistemas de almacenamiento para el transporte. Esta categoría incluye todos los dispositivos móviles utilizados para colocar y transportar mercancías en los vehículos y, finalmente, para la carga / descarga (tales como remolques para el transporte de largo recorrido). 
- Vehículos de tracción no convencional.

\subsection{Generalidades sobre la problemática de la logística urbana}

Algunos problemas comunes relacionados con el transporte de mercancías en ámbito urbano que pueden citarse son:

- La estructura urbana y el uso del suelo de las ciudades, típico de los centros históricos de origen antiguo. Muchas ciudades europeas tienen orígenes antiguos y calles estrechas que no son aptas para el tráfico de vehículos ni para las operaciones de carga y descarga. La mayoría de los centros históricos son zonas comerciales muy activas donde la adopción de procesos de logística urbana adecuados sigue siendo un gran desafío.

- La falta de planificación relativa a la logística urbana: Generalmente los aspectos relacionados con las actividades de logística urbana no se consideran como una prioridad en la planificación de la movilidad. Se percibe como un elemento marginal, sobre todo por el predominio del sector privado. Hasta ahora, el número de ciudades que ha desarrollado un "Plan de Logística Urbana Sostenible" es muy limitado.

- La falta de fondos para los municipios para desarrollar políticas adecuadas para resolver este problema es otro tema relevante.

El sector de la logística urbana no puede considerarse particularmente avanzado desde el punto de vista tecnológico y organizativo. Por lo tanto, la investigación y la innovación tecnológica pueden desempeñar un papel importante en la mejora de la competitividad de este sector y podrían ayudar al desarrollo de la economía regional, dando beneficios adicionales:

- Las nuevas tecnologías y productos que podrían ampliar el mercado para un número significativo de empresas proveedoras de equipos y soluciones para la logística urbana.

- La competitividad económica del sector industrial local se puede aumentar a través de un mayor nivel de innovación.

Todas las regiones asociadas DOROTHY se enfrentan a los mismos problemas: tienen que hacer frente a los retos de la globalización. El aumento de su capacidad de investigación e innovación es sin duda una elemento prioritario para lograr el éxito.

Desde un punto de vista regional, las necesidades más relevantes que se han puesto de manifiesto son:

- La coordinación de las políticas locales, con el fin de armonizar el marco regulatorio de la logística urbana en el territorio de acuerdo con una estrategia general.

- La definición de una política regional que permita la asignación de los fondos para dinamizar el sector. 


\section{EL JOINT ACTION PLAN}

De acuerdo con la el Programa de la CE de Regiones del Conocimiento, un plan de acción conjunta (JAP) es un instrumento capaz de impulsar el desarrollo económico a través de actividades de investigación y desarrollo tecnológico en un tema o sector económico y mejorar toda la cadena de valor añadido de la innovación.

Durante el proyecto DOROTHY más de 100 entidades (empresas, universidades, centros de investigación, instituciones públicas y privadas y asociaciones del sector han estado involucrados en el diseño del JAP. Durante el diseño del JAP se analizaron los siguientes aspectos:

- El nivel de desarrollo de la logística urbana y la estructura del mercado.

- Las características de la oferta y la demanda de servicios y productos.

- Las actividades de las empresas que operan en todos los segmentos de este mercado y su oferta.

- El trabajo desarrollado por las universidades y centros de investigación que trabajan en el campo de la logística urbana.

- Los programas de investigación y las agendas de innovación regionales.

A partir del análisis llevado a cabo desde el inicio del proyecto, se definieron las actividades de innovación específica y áreas de investigación potencialmente interesantes. Este análisis se centró en la especialización local de cada clúster y su potencial, las condiciones locales y el potencial de la demanda local para la innovación, la posibilidad de explotar la innovación fuera del ámbito territorial, a nivel nacional e internacional.

Desde el punto de vista tecnológico, los principales sectores que han sido considerados para ser incluidos en el JAP fueron:

- Los sistemas de TIC, tanto de apoyo de los operadores logísticos y como a las ciudades para la implementación de esquemas de regulación y control de accesos.

- Vehículos innovadores (con especial atención a los vehículos eléctricos) y el equipos de carga / descarga, almacenamiento y sistemas de distribución.

- Nuevos esquemas de distribución para el entorno urbano y sus sistemas de apoyo.

Además, se tuvo en cuenta el marco económico y legislativo, así como beneficios sociales, ambientales y urbanos esperados.

Cabe destacar el objetivo general del JAP es la mejora de la logística urbana en las regiones a través de:

- La constitución de clústeres capaces de incrementar el nivel de Innovación

- El fortalecimiento de la cooperación entre los agentes del sector.

- El establecimiento de relaciones estables de cooperación entre los clústeres. 


\subsection{Impactos esperados.}

El proyecto Dorothy ha incluido también un análisis de los impactos esperados tras la aplicación de las mejoras propuestas. Estos impactos son, principalmente, los siguientes:

- Económico: a una mayor competitividad de las industrias del sector que operan en todos los niveles del mercado (operadores de logística urbana, proveedores de tecnología, ingeniería / consultores) con las implicaciones laborales pertinentes a través de:

○ Innovación: fortalecimiento de las relaciones entre las universidades, institutos de investigación, empresas tecnológicas y operadores logísticos.

- Mayor calidad y menor tiempo de comercialización: cooperación entre empresas con intereses comunes o complementarios dentro de los clústeres regionales.

- Acceso más sencillo a fondos europeos para la innovación.

- Políticas regionales más comprometidas regionales con la innovación.

- Ampliación de mercado: la cooperación con empresas de otras regiones europeas y el apoyo a la internacionalización.

- Medio Ambiente: mejora del entorno urbano mediante la reducción de los efectos negativos de la logística en la ciudad.

- Organización: mediante la mejora de los procesos con la aplicación de más y mejores tecnologías y sistemas de información.

- Social: Alcanzar un consenso en el sector sobre los esquemas y soluciones de logística urbana para lograr una distribución justa de los costes y beneficios.

- Urbano: mejorar la organización y el uso de los espacios urbanos limitando las interferencias con otras actividades urbanas.

\subsection{Las acciones del JAP}

Cada acción ha sido descrita según una plantilla estándar que contiene:

- Un resumen.

- Objetivos.

- Descripción de las condiciones generales en las que se enmarca la actuación y su relación con el sector (en las regiones o en general en Europa).

- Descripción de la acción propiamente dicha.

- Evaluación del impacto a nivel normativo, económico, ambiental, social y urbano.

- Potencialidades y barreras

- Plan de financiación para la ejecución de la acción

- Referencias.

Las acciones que conforman el JAP son las siguientes: 
1.1. Planes de Logística Urbana Sostenible (iniciales en inglés, SULPs)

1.2. Políticas, Regulación de la Logística Urbana and Tecnologías de apoyo

2.1. Índice de Rendimiento de la Logística Urbana

2.2. Disponibilidad de Open Data para la logística urbana

2.3. Sistema de Acreditación Regional de Logística Urbana

3.1. Soluciones innovadoras de TIC para apoyar esquemas de regulación avanzados en la Logística Urbana

3.2. Arquitecturas de Open Data de apoyo a la Logística Urbana

3.3. Áreas de entrega de proximidad (Proximity Delivery Areas)

3.4. MoU - Marco de Cooperación entre los Clúster del Proyecto Dorothy.

3.5. Fortalecimiento de la colaboración de los Clústeres.

4.1. Titulación de Master International en Logística Urbana

4.2. Formación del personal en nuevos esquemas y técnicas.

5.1. Apoyo al uso de "Cargo bike" para la entrega de mercancías en área urbana

5.2. Organización de una red de operadores en las ciudades para la puesta en marcha de puntos de proximidad de entrega para paquetería.

6.1. Creación de una plataforma web para mejorar las relaciones entre los Clústeres de Logística Urbana.

6.2. Cooperación entre los Centros de Investigación y Universidades de las regiones para desarrollar líneas comunes de investigación.

6.3. Observatorio de la logística urbana

7.1. Catálogo de los Clústeres Regionales

7.2. Acción hacia países no europeos.

7.3. Coordinación con iniciativas regionales existentes

7.4. Seminario Internacional en Medellín.

A continuación se recoge un breve resumen sobre los contenidos cada una de las acciones.

\subsubsection{Planes de Logística Urbana Sostenible (iniciales en inglés, SULPs)}

La acción se centra en el desarrollo de incentivos de los gobiernos nacionales y regionales para la implementación de los Planes de Logística Urbana Sostenible (SULPs) para apoyar a los actores clave en la toma de decisiones a nivel local con el fin de impulsar buenas prácticas en la distribución urbana dentro del área regional, y para mejorar los procesos de distribución de mercancías buscando el cumplimiento de los objetivos de desarrollo económico, consumo de energía, respeto por el medio ambiente y eficiencia.

Un SULP es una parte esencial de un Plan de Movilidad Urbana Sostenible (PMUS) y su objetivo es abordar el tema de la logística urbana desde una perspectiva integral. El SULP se dirige a los grupos de interés, con un enfoque especial en las autoridades locales, para definir estrategias, medidas y regulaciones con el fin de aumentar la sostenibilidad global de la logística urbana, es decir, conseguir un menor consumo de energía, menos congestión en 
las zonas urbanas, menos contaminación del aire, menos ruido y menos emisiones de gases de efecto invernadero con la consiguiente mejora de la calidad de vida urbana.

La difusión de los SULPs también tiene efectos beneficiosos para los Clústeres, ya que:

- Incentiva a las ciudades a adoptar regulaciones innovadoras para la distribución de mercancías.

- Aumenta el mercado para las empresas de consultoría e ingeniería

\subsubsection{Políticas, Regulación de la Logística Urbana and Tecnologías de apoyo}

Esta acción tiene como objetivo crear una herramienta dirigida a los municipios y otras autoridades locales para facilitar el proceso de toma de decisiones sobre la logística urbana. De hecho, las ciudades europeas se ven obligadas a hacer frente a una amplia gama de problemas relacionados con la distribución urbana de mercancías pero, mientras que el transporte urbano es foco de atención, la logística urbana suele ser un tema marginal pese a su importante impacto directo tanto económico como ambiental sobre las ciudades. La importancia de este tema también está atestiguada por el hecho de que el Libro Blanco de Políticas del Transporte de la CE estableció como uno de los 10 objetivos a alcanzar para el año 2030 lograr una log 'sitica urbana libre de emisiones de $\mathrm{CO}_{2}$.

Uno de los principales problemas encontrados en las ciudades es el bajo nivel de conocimiento acerca de cómo las soluciones innovadoras pueden contribuir a mejorar el estado de la logística urbana. Por esta razón, surge la necesidad de realizar un esfuerzo de difusión de conocimiento como pre-requisito para la introducción de dichas soluciones.

Esta acción plantea como herramienta la creación de un "catálogo" de políticas de regulación, medidas de organización y tecnologías de apoyo. El catálogo debe favorecer la puesta en marcha de soluciones innovadoras y podría incluir, además, las evaluaciones del perfil urbano de las ciudades para el establecimiento de soluciones a medida para maximizar los beneficios económicos, sociales y medioambientales.

\subsection{3. Índice de Rendimiento de la Logística Urbana}

El objetivo principal de esta acción es desarrollar un modelo de índice y patrones de referencia para la logística urbana, lo que permitiría la evaluación del nivel de rendimiento de la logística urbana en una ciudad y realizar comparaciones con otras ciudades o regiones.

Este índice de rendimiento de la logística urbana será una valiosa herramienta de guía que ayude a las autoridades a tomar decisiones estratégicas sobre las políticas urbanas y de logística y definir las acciones a implementar. Para las entidades privadas, el índice proporcionará una herramienta de orientación para evaluar la competitividad. Además, proporcionará información a los operadores logísticos sobre los principales obstáculos en 
cada ciudad. Otra utilizad será la de facilitar la puesta en marcha de soluciones, identificando las ciudades más "amigables" en términos de logística urbana y proporcionando información a los productores de soluciones y tecnologías sobre las necesidades locales clave.

El índice de rendimiento también será importante para las empresas de asesoramiento y planificación, como herramienta de diagnóstico. El índice de rendimiento permitirá la identificación de las áreas críticas de intervención para que se pueda crear soluciones tecnológicas a medida, por lo que facilitará el mercado a las empresas tecnológicas.

\subsubsection{Disponibilidad de Open Data para la logística urbana}

El objetivo de esta acción es estimular la recopilación y publicación de datos en abierto (en inglés, Open Data) valiosos y útiles para la logística urbana. Con este fin, la acción establecerá las actividades preliminares necesarias para definir y recoger datos completos, fiables, estandarizados y útiles. Estos datos estarán disponibles para el público en general.

En el marco de esta acción, la idea es utilizar estos datos para alimentar a una plataforma urbana de Logística Open Data (ULODaP), objeto de la acción 3.2, y al sistema de acreditación regional, objeto de la acción 2.3., como primeros ejemplos de uso real. Sin embargo, se pretende que estos datos estén disponibles para todo el mundo para favorecer el desarrollo de nuevas aplicaciones.

La logística urbana es una parte específica de la demanda de transporte y en lo relativo al análisis de datos relacionado con la última milla existe una carencia de datos que aún no ha sido objeto de análisis, estudio y optimización como se hace con la movilidad de pasajeros.

La acción también estudiará algunas actuaciones a poner en marcha por las regiones, para fomentar o incentivar a las ciudades para difundir datos abiertos de acuerdo con las directrices definidas por esta acción.

\subsubsection{Sistema de Acreditación Regional de Logística Urbana}

El propósito de esta acción es animar a las autoridades regionales a poner en práctica un proceso de acreditación, promoviendo el intercambio de experiencias entre las regiones y la armonización de los procedimientos y reglamentos, con el objetivo de utilizar las sinergias para mejorar la logística urbana y lograr un impacto positivo en la calidad de vida.

Para asegurar el éxito de esta acción, se propone el desarrollo de una "nube" o plataforma a web, el Sistema de Logística Regional y Urbana de Acreditación (Rulas) que proporcione la sostenibilidad al proceso y que esté disponible para todas las entidades involucradas.

Debido a las singularidades de cada región, debe nombrarse a un coordinador regional con 
la formación adecuada. Los operadores logísticos urbanos y todas las partes interesadas deben ser escuchados con el fin de armonizar los procedimientos tanto como sea posible, teniendo en cuenta las características específicas de cada región.

Esta acción debería desarrollarse en estrecha coordinación con las autoridades reguladoras. Se espera que haya importantes retornos económicos, ambientales y de eficiencia, y un incremento de calidad y eficiencia en los servicios prestados por las autoridades municipales. Los posibles retornos deben exceder en gran medida las inversiones previstas, así como generar impactos positivos significativos sobre el medio ambiente y el turismo. Por último, pero no menos importante, la colaboración y armonización del trabajo debería favorecer el intercambio de experiencia y la puesta en marcha de nuevos proyectos entre las regiones europeas.

\subsubsection{Soluciones innovadoras de TIC para apoyar esquemas de regulación avanzados en la Logística Urbana}

Esta acción está dedicada a la implementación de una plataforma TIC para gestionar los esquemas de regulación de la logística urbana en las ciudades, especialmente centrados en las áreas de carga y descarga y el control de accesos. Se tiene en cuenta la posibilidad de gestionar la demanda de plazas de aparcamiento.

La descripción de la acción considera aspectos normativos y tecnológicos. También se describen los requisitos tecnológicos generales para implementar una nueva plataforma de gestión con el objetivo de mejorar la gestión de los espacios de carga y carga, teniendo en cuenta la relación entre la oferta y la demanda.

\subsubsection{Arquitecturas de Open Data de apoyo a la Logística Urbana}

El objetivo de esta acción es estimular nuevos servicios, mejores y más integrados de logística urbana, para las autoridades públicas y los operadores del sector, de acuerdo con las tendencias propuestas a nivel europeo en los SULP y utilizando el potencial de los datos en abierto que todavía no se aplican.

Para este objetivo, se propone desarrollar una Plataforma de Open Data para la Logística Urbana Sostenible (de las siglas en inglés, ULODaP), que sea un centro capaz de recoger el conjunto de datos útiles para las operaciones de logística urbana y que proporcione servicios a autoridades y operadores. La forma de compartir esos datos debe estandarizarse a nivel (inter) regional.

Esta acción es estrictamente complementaria a la acción " Disponibilidad de Open Data para la logística urbana ", ya que pretende garantizar la disponibilidad de datos abiertos desde las 
administraciones públicas. Por otra parte, también puede estar relacionado con la acción de "sistema de acreditación regional " que puede ser una aplicación específica de esta plataforma general.

Para el éxito de esta acción se debe contar con el apoyo de las autoridades reguladoras, a nivel local, regional y nacional.

\subsection{8. Áreas de entrega de proximidad (Proximity Delivery Areas)}

Las áreas de entrega de proximidad (PDA) son básicamente plataformas de transbordo urbanas, donde es posible proporcionar asistencia para la clasificación de la paquetería para la última milla dirigida al comercio / consumidores ubicados en áreas próximas (a menudo el centro de la ciudad). Las mercancías se descargarían de los vehículos entrantes, y se repartirían con vehículos más respetuosos con el medio ambiente, como eléctricos y bicicletas, para el segmento final de la distribución. Las PDAs se pueden desarrollar en diferentes formas, tamaños y modalidades de funcionamiento, de acuerdo con las limitaciones urbanas específicas, tales como la morfología, distribución de actividades y el volumen de las mercancías entrantes. Esta flexibilidad puede fomentar el su uso para gestionar una gran parte de las mercancías dirigidas a la ciudad, lo que reduciría los problemas de congestión que afectan a dichas áreas.

Esta acción está dedicada al desarrollo de productos de PDA y su difusión. También sugiere una estrategia y modalidades operativas para implementar una PDA zonas de tráfico limitado, alrededor de los centros históricos de las ciudades y otros entornos urbanos.

\subsubsection{MoU - Marco de Cooperación entre los Clúster del Proyecto Dorothy.}

El proyecto DOROTHY ha conseguido el resultado de la creación de tres Clústeres regionales de innovación en Valencia - España, Lisboa - Portugal y Craiova - Rumania bajo la tutorización del Clúster Toscana ya existente.

Para garantizar que este logro sea duradero en el tiempo se ha establecido un marco de cooperación entre los 4 clústeres que permanecerá después del final del proyecto. Por esta razón, entre otras actividades llevadas a cabo por el proyecto, se elaboró un Memorando de Entendimiento (con la siglas en inglés, MoU) que ha sido firmado por los 4 Clústeres en forma de un acuerdo mutuo y multilateral entre ellos.

El memorando contiene un conjunto de acciones específicas que se llevarán a cabo en el marco del JAP y una serie de propuestas sobre áreas temáticas específicas que se abordarán mediante la cooperación entre los clústeres.

\subsubsection{Fortalecimiento de la colaboración de los Clústeres.}


Esta acción supone la puesta en marcha de actividades que refuerzan la cooperación entre los Clústeres de Dorothy con el fin de aprovechar las oportunidades de crecimiento, maximizando las sinergias y acelerar la convergencia económica regional a través de los diferentes socios en las regiones, en línea con las prioridades similares en el campo de la logística urbana. Esto se logra a través de una identificación común y la realización de actividades e iniciativas conjuntas con el fin de facilitar el establecimiento de inversiones en innovación y el aumento de la productividad, mediante la identificación de soluciones y retos de I + D, vínculos en la cadena de valor, oportunidades de negocio para la colaboración entre los países y sectores, en particular entre las Pymes.

Esta acción tiene el objetivo final de facilitar la innovación, principalmente a través de la futura puesta en marcha de consorcios de investigación para los programas europeos e internacionales dedicados a la financiación de la I + D o de otras iniciativas, así como el fortalecimiento de la competitividad de las empresas de los clústeres.

\subsubsection{Titulación de Master International en Logística Urbana}

El objetivo principal de esta acción es diseñar y preparar el lanzamiento de un grado de master de alto nivel internacional sobre logística urbana. El máster sería reconocido por las universidades e instituciones de investigación de las regiones involucradas, como la Universidad Politécnica de Valencia (UPV), Universitatea din Craiova, Instituto Pedro Nunes, Associacao para a Inovacao e Desenvolvimento em ciencia e tecnología y Universidad de Florencia, así como otras instituciones interesadas. Sus estudiantes tendrían que ser titulados universitarios y su duración debe ser de entre 60-120 créditos ECTS.

Uno de los objetivos principales es que el grado tenga un carácter inter-universitario. Este objetivo se lograría a través de la participación de varias universidades o entidades de educación superior. Esta cooperación será uno de los primeros resultados del acuerdo de cooperación entre las universidades previstos por esta acción del JAP.

\subsubsection{Formación del personal en nuevos esquemas y técnicas.}

Esta acción es una respuesta a la necesidad de especialización por parte de las empresas pertenecientes a los clústeres y de otras empresas relacionadas con la logística urbana, para mantener un nivel de cualificación alta entre sus empleados y por ser capaces de innovar. Las empresas, en el desarrollo de sus líneas de productos / servicios, a menudo necesitan aumentar sus competencias sobre temas específicos con una cantidad limitada de recursos. En particular, la aplicación de las medidas contempladas en el JAP frecuentemente involucra la introducción de nuevas tecnologías para la aplicación de nuevos esquemas y técnicas a las que actualmente se utilizan para la distribución urbana de mercancías. 
La acción de formación del personal sobre temas innovadores se centra en aumentar el conocimiento y la competencia general de los actores implicados (operadores logísticos, proveedores de tecnología, el personal de los consejos de administración, las administraciones locales que están a cargo de los servicios de logística urbanas y otras partes interesadas) en nuevas tecnologías y técnicas.

Esta acción está dirigida a proporcionar a los clústeres la capacidad de desarrollar la educación profesional continua dirigida a la innovación. Esto significa que los clústeres deben establecer mecanismos adecuados capaces de identificar las necesidades de formación y organizar eventos de capacitación que pueden ser muy diferentes en los temas, los beneficiarios y las metodologías.

\subsubsection{Apoyo al uso de "Cargo bike" para la entrega de mercancías en área urbana}

Esta acción está dedicada a promover el uso de bicicletas de carga (in inglés, cargo-bikes) en la logística urbana. Para ello, se ha considerado el marco regulatorio y la infraestructura.

El uso de bicicletas de carga tiene como objetivo garantizar un servicio de entrega regular dirigido a las actividades comerciales ubicadas en las zonas urbanas, especialmente en los centros de las ciudades, de una manera más sostenible. La acción es factible para las ciudades de tamaño grande y medio y extensas áreas urbanas, donde un marco regulador adecuado puede estimular el uso de las bicicletas de carga para el transporte urbano de mercancías.

\subsubsection{Organización de una red de operadores en las ciudades para la puesta en marcha de puntos de proximidad de entrega para paquetería.}

Esta acción tiene como objetivo definir un esquema de organización e identificar medidas e incentivos para la última milla y la entrega. La idea es implementar un sistema fácil y económico, basado en las necesidades de las ciudades, de los consumidores y de los operadores, que pueda ser adoptado por las ciudades de la misma forma para la entrega de paquetería en los centros de las ciudades.

El sistema consiste en dotar a los lugares adecuados, es decir, pequeños negocios como farmacias, tintorerías, cafeterías, kioscos, con una pequeña área de almacenamiento donde los conductores pueden dejar los paquetes para que los clientes los puedan recoger.

El uso de este tipo de punto de entrega de proximidad supone un ahorro de costes para los operadores logísticos, en términos de un menor número de entregas sucesivas en las casas, y para la ciudad, debido a la reducción en el número de vehículos circulando.

\subsubsection{Creación de una plataforma web para mejorar las relaciones entre los Clústeres}




\section{de Logística Urbana.}

La plataforma de matchmaking es una herramienta para facilitar las relaciones y contribuir al desarrollo de una nueva cultura y una nueva planificación de negocios, con el fin de organizar eventos de colaboración y networking para las empresas y las instituciones de investigación.

La acción fija como objetivos las pequeñas empresas del sector (preferentemente las TIC), grupos de investigación y administraciones públicas. Las interacciones de estos objetivos se harán a través de eventos con diferentes escenarios: B2B, B2R para facilitar la construcción de proyectos de innovación; R2R para crear trayectorias de la innovación y la innovación de formación; B2I para escuchar las necesidades del territorio y poner en práctica nuevas políticas; y R2I para crear un puente entre la innovación y el territorio.

Esta plataforma será una herramienta básica para apoyar y mejorar la cooperación entre los Clústeres regionales y sus empresas.

\subsubsection{Cooperación entre los Centros de Investigación y Universidades de las regiones para desarrollar líneas comunes de investigación.}

El objetivo principal de los Clústeres de Dorothy es ampliar al máximo la cooperación entre las cuatro regiones, los centros de investigación y los propios Clústeres regionales impulsando la investigación en beneficio de las Pymes y de la competitividad regional y el crecimiento global. Con esta acción concreta en este sentido, las universidades y organismos de investigación que son miembros de los Clústeres de DOROTHY se comprometen a fortalecer su cooperación en el campo de la logística urbana con el fin de apoyar el desarrollo de los clústeres. Como primer paso en esta dirección se van a establecer áreas específicas de interés común para la cooperación.

\subsubsection{Observatorio de la logística urbana}

El objetivo de esta acción es el diseño de un observatorio de la logística urbana con el objetivo, en una primera fase, de recopilar y evaluar buenas prácticas y acciones llevadas a cabo para realizar una 'auto-evaluación comparativa ". En el futuro se pretende lograr una base de datos numéricos destinados a la evaluación cuantitativa y la evaluación comparativa.

Este observatorio facilita a un nivel de los clústeres y de las acciones - experiencias piloto y soluciones implementadas de forma genérica, así como las buenas prácticas - el intercambio de información, conocimientos y experiencias y la evaluación comparativa. También puede ayudar a definir los objetivos y contenidos de investigación y formación en el campo de la logística urbana. 
La acción tendrá un papel de facilitador para proporcionar información y para estimular la participación de las partes interesadas - operadores, proveedores de tecnología, instituciones de investigación y desarrollo, entidades públicas y privadas, etc. - que trabajan en el campo de la logística urbana, así como en otras disciplinas relacionadas, incluido el desarrollo urbano y regional, la planificación urbana, el transporte, la energía y las ciencias ambientales. También se dirige a los beneficiarios de la logística urbana, principalmente ventas al por mayor y los usuarios finales, así como también a las entidades reguladoras y los responsables de tráfico y el espacio urbano.

El observatorio también constituirá una plataforma de recursos para varias herramientas desarrolladas dentro y fuera del proyecto DOROTHY, como por ejemplo un catálogo de buenas prácticas, iniciativas, materiales de capacitación, fotos, videos y difusión de las oportunidades de financiación de la UE.

\subsubsection{Catálogo de los Clústeres Regionales}

El objetivo de esta acción es la realización y promoción en los Clústeres Regionales de un catálogo concebido para la difusión de las empresas y entidades que los componen en.

El catálogo tiene un papel importante para la difusión del concepto de Clúster en el campo de la logística urbana, la promoción de la imagen de sus miembros, la mejora de las posibilidades de crear asociaciones (empresariales, de investigación e innovación, desarrollo regional, etc.) y el apoyo a las posibilidades de cooperación para la internacionalización.

\subsubsection{Acción hacia países no europeos.}

Esta acción está dedicada a apoyar la internacionalización de las empresas de los clústeres de Dorothy. Después de haber analizado el mercado existente, la red y sus socios potenciales, se han identificado algunos países no europeos como más atractivos para desarrollar la estrategia internacional en el marco de Dorothy. La acción más importante para apoyar la internacionalización de los grupos DOROTHY es una iniciativa dirigida a México.

\subsubsection{Coordinación con iniciativas regionales existentes}

La internacionalización es un conductor para el desarrollo de negocio. La internacionalización y la actividad empresarial internacional entre las Pymes es un tema de considerable importancia, debido principalmente a los efectos de crecimiento observados de las empresas transnacionales y la capacidad demostrada de las Pymes para impulsar el desarrollo económico a nivel nacional, regional y global.

Esta acción tiene por objeto incluir a los Clústeres Dorothy en algunas iniciativas ya 
previstas para el apoyo a la internacionalización de las empresas por las regiones o por cámaras de comercio locales.

\subsubsection{Seminario Internacional en Colombia.}

Esta actuación consiste en la organización de un Seminario Internacional sobre la logística urbana en la ciudad de Medellín (Colombia). Este seminario será un evento internacional con el objetivo de compartir experiencias sobre la logística urbana en Europa y Colombia, con una posible extensión a otros países de América Latina.

\section{GENERALIDADES DEL MARCO NORMATIVO.}

A nivel europeo, no existe una legislación específica sobre la logística urbana, pero hay muchas recomendaciones y directrices. En este apartado se referencian las más significativas en relación con las acciones del JAP.

La Comisión Europea publicó en 1992 el Libro Blanco sobre la política común de transportes, dedicado fundamentalmente a la apertura del mercado. Casi diez años después, el Libro Blanco de 2001 hizo hincapié en la necesidad de gestionar el crecimiento del transporte de una manera más sostenible con un uso más equilibrado de los modos de transporte. En 2011, el Libro Blanco sobre el transporte y el programa Horizonte 2020 fijaron objetivos ambiciosos para la reducción de emisiones de $\mathrm{CO} 2$ relacionadas con la logística de la ciudad. El objetivo es lograr que logística de la ciudad esté libre de emisiones de $\mathrm{CO} 2$ en los principales centros urbanos en 2030.

La Comisión Europea ha adoptado un plan de trabajo con 40 iniciativas concretas para la próxima década de cara a construir un sistema de transporte competitivo, reduciendo drásticamente la dependencia de Europa del petróleo y las emisiones de carbono. En relación con el JAP las iniciativas relacionadas son las que hacen referencia a los siguientes aspectos:

- Transporte de mercancías por carretera

- Transporte multimodal de mercancías

- Hoja de ruta tecnológica

- Marco regulatorio para la innovación en el transporte

- Etiquetado de los vehículos según sus emisiones de CO2 y eficiencia energética

- Cálculo de la huella de carbono

- Eco-conducción y límites de velocidad

- Planes de movilidad urbana

- Estrategia para acercarse a las emisiones 0 por la logística urbana en 2030.

Los principales grupos de interés para todas las regiones en cuanto a la aplicación de la regulación de la logística urbana son los municipios. Por supuesto, tienen que trabajar en el 
marco fijado por las directivas regionales y las leyes nacionales, pero tienen un papel fundamental en la definición de todos los esquemas de regulación para la distribución de mercancías en su territorio.

El JAP representa un paso adelante en el fomento de la adopción de políticas de regulación innovadoras en las regiones. Las medidas contenidas en el JAP están sujetas al marco normativo local, por lo que en algunos casos puede que tengan que ser adaptados a nivel regional para maximizar sus posibilidades de éxito o simplemente para hacerlas aplicables.

La aplicación con éxito de algunas acciones importantes del JAP podría contribuir a la consecución de algunos de los objetivos que figuran en las recomendaciones de la UE. En particular, el tema específico de la armonización de la legislación y normas en el campo de la logística urbana podría recibir alimentarse significativamente de algunas de las acciones del JAP. De hecho, se plantea lograr una revisión de los reglamentos y normas existentes y una cierta armonización de los procedimientos entre las regiones asociadas que podrían ser transferidas a otras regiones europeas.

\section{INTEGRACIÓN DEL JAP EN LAS POLÍTICAS REGIONALES}

El JAP es un ambicioso programa para apoyar el desarrollo económico y social de las regiones de una manera territorialmente equilibrada y sostenible en el campo de la logística urbana.

La logística urbana se ha identificado como un importante problema social y urbano, ya que la gran mayoría de las ciudades se ven afectadas directamente, pero al mismo tiempo puede ser un elemento importante del desarrollo económico.

El proyecto DOROTHY ha identificado un conjunto de acciones coordinadas, recogidas en el JAP; y ha definido los recursos financieros necesarios para su implementación.

Cuando se trata de programas tan complejos y articulados como el que se define con el JAP, es muy importante crear sinergias entre las diferentes fuentes de financiación: ESIF, Horizonte 2020 y otros programas de investigación e innovación de la Unión Europea relacionadas con la competitividad.

Otro punto muy importante es tener en cuenta todas las posibles sinergias con las Estrategias Regionales para la especialización inteligente. Se plantean algunas sinergias a través del uso de Horizonte 2020 y los Fondos de Inversión Estructural (ESIF) así como mediante la coordinación de proyectos paralelos que se complementan entre sí y son financiados por diferentes fuentes.

Las posibles fuentes de financiación para el JAP están vinculadas principalmente a los 
fondos europeos y nacionales que se gestionan a través de las regiones, como los Fondos Estructurales y a programas europeos lanzados y gestionados por la Unión Europea.

El documento del JAP realiza un análisis sobre las posibilidades de financiación en cada región en relación con cada una de las acciones.

\section{AGRADECIMIENTOS}

A los autores nos gustaría incluir un especial agradecimiento a todos el consorcio Dorothy, por su entregado trabajo, y también a aquellas empresas y entidades que ha colaborado de forma desinteresada para hacer posible la creación del JAP.

\section{REFERENCIAS}

La redacción de este artículo se ha basado en lo recogido en JAP del proyecto Dorothy, disponible para su descarga en www.clusterdorothy.com

Otras referencias utilizadas:

EUROPEAN COMMISSION 2011 Transport White Paper - Roadmap to a Single European Transport Area - Towards a competitive and resource efficient transport system, http://ec.europa.eu/transport/themes/strategies/2011_white_paper_en.htm, 28-3-2011 